DOI: $10.3901 / J M E .2020 .24 .125$

\title{
四轮轮毂电机驱动电动汽车电液复合 制动平顺性控制策略"
}

\author{
张 雷 1,2 刘青松 ${ }^{1,2}$ 王震坡 ${ }^{1,2}$
}

(1. 北京理工大学北京电动车辆协同创新中心 北京 100081 ;

2. 北京理工大学电动车辆国家工程实验室 北京 100081)

摘要: 液压制动与电机再生制动的时域响应差异导致电动汽车在制动模式切换时产生冲击感, 影响驾驶员驾驶感受和乘坐舒 适性。以四轮轮㪍电机驱动电动汽车为研究对象, 提出一种基于分层架构的电液复合制动平顺性控制策略。针对 “高压蓄能 器+电机原” 式电子液压制动系统(EHB), 上层控制器提出基于模糊控制的轮缸压力控制策略; 针对制动模式切换过程中产生 的冲击, 下层控制器提出包括液压介入预测模块和电机制动补偿模块的电液复合制动平顺性控制策略。通过 Simulink-AMESim 联合仿真平台进行仿真试验验证。结果表明, 轮缸压力控制策略能够保证轮缸液压力较好地追随目标压力, 且稳态误差不超过 $2 \%$; 电液复合制动平顺性控制策略能够有效提高制动系统的响应速度，同时显著降低制动模式切换时的 冲击, 能提升车辆制动平顺性和乘坐舒适性。

关键词：电液复合制动；轮缸压力控制；模糊控制；平顺性控制策略

中图分类号: TG156

\section{Electro-hydraulic Brake Control for Improved Ride Comfort in Four-wheel-independently-actuated Electric Vehicles}

\author{
ZHANG Lei $^{1,2}$ LIU Qingsong ${ }^{1,2}$ WANG Zhenpo ${ }^{1,2}$ \\ (1. Collaborative Innovation Center for Electric Vehicles in Beijing, Beijing Institute of \\ Technology, Beijing 100081; \\ 2. National Engineering Laboratory for Electric Vehicles, Beijing Institute \\ of Technology, Beijing 100081)
}

\begin{abstract}
The time domain response difference between the hydraulic and the electric brake often causes shocks during the brake mode switching process in between for electric vehicles (EVs), which would significantly compromise ride comfort. A ride-comfort-enabled strategy that consists of an upper and a lower controller is proposed for electro-hydraulic-combined brake system in a four-wheel-independently-actuated electric vehicle (FWIA EV). In the upper controller, a wheel cylinder pressure control strategy based on fuzzy control is proposed for an electro-hydraulic brake system (EHB) composed of a high-pressure accumulator and a motor pump. In order to tackle with the impact caused by brake mode switching, a ride-comfort-enabled strategy is proposed in the lower layer. It incorporates a hydraulic intervention prediction module and an electric brake compensation module. Finally, the proposed strategy is examined under various brake scenarios in Simulink-AMESim joint simulation. The results show that the wheel cylinder pressure can precisely track the target pressure with a steady-state error of less than $2 \%$ under different brake demands. The brake response is guaranteed and the impact during the brake mode switching process is significantly reduced under the proposed control strategy, thus achieving improved brake performance and ride comfort.
\end{abstract}

Key words: electro-hydraulic brake; wheel cylinder pressure control; fuzzy control; ride-comfort-enabled strategy

* 国家重点研发计划 (2017YFB0103600)和北京市科技新星计划 (Z201100006820007)资助项目。20200324 收到初稿, 20201014 收到修改稿 


\section{0 前言}

汽车电动化、网联化、智能化是提高交通系统 安全性和实现节能减排的重要手段, 已成为世界各 国的普遍共识。电子液压制动系统(Electro-hydraulic brake system, EHB)通过电子控制系统控制液压执行 机构实现与制动踏板的机械解耦, 可在驾驶员未介 入时实现主动制动功能, 为实现制动能量回收功能 以及主动紧急制动、自适应巡航等高级驾驶辅助功 能提供了基础。四轮轮毂电机驱动电动汽车将四个 轮毂电机分别安装在轮辋内部, 具有车身布置灵活、 结构紧凑、易于实现底盘模块化设计和主动控制等 优点, 成为新能源汽车的重要技术发展方向之一。 由于四个轮毂电机均可独立控制, 与 EHB 构成电液 复合制动系统, 可实现单轮制动力解耦控制 ${ }^{[1]}$, 不 仅可以大幅提高制动能量回收率, 还能显著提高紧 急工况下车辆的横向、纵向稳定性 ${ }^{[2-3]}$ 。

根据建压方式的不同, EHB 主要包括三种类型, 即 “主缸助力电机+减速机构” 式、“高压蓄能器+液 压泵” 式和电机柱塞泵式 ${ }^{[4]}$ 。余卓平等 ${ }^{[5]}$ 总结了三种 EHB 的优缺点及其主缸、轮缸压力控制方法。相较于 其他两种类型, “高压蓄能器+液压葲” 式 EHB 具有 体积小、高压源稳定等特点, 可通过控制液压执行单 元的电磁阀实现四个轮缸压力的独立、精确控制, 简 化了系统结构。本文采用“高压蓄能器+液压泵”式 $\mathrm{EHB}$ 与轮毂电机构成电液复合制动系统。

“高压蓄能器+电机泵”式 EHB 轮缸压力控制实 质上是电磁阀控制。针对轮缸压力控制, $\mathrm{CHOI}$ 等 ${ }^{[6]}$ 提出了一种线性流量控制(Linear flow control, LFC) 方法, 具体控制流程如图 1 所示。该方法通过控制 脉宽调制(Pulse width modulation, PWM)信号的占空 比调节电磁阀的线圈电流, 从而实现轮缸压力的线 性调节。在此基础上, 考虑目标压力变化率、线性 阀前后压差以及当前轮缸压力, 王祥 ${ }^{[7]}$ 提出了一种 基于 BP 人工神经网络的控制方法, 具体控制逻辑 如图 2 所示。刘刚等 ${ }^{[8]}$ 基于建立的回路

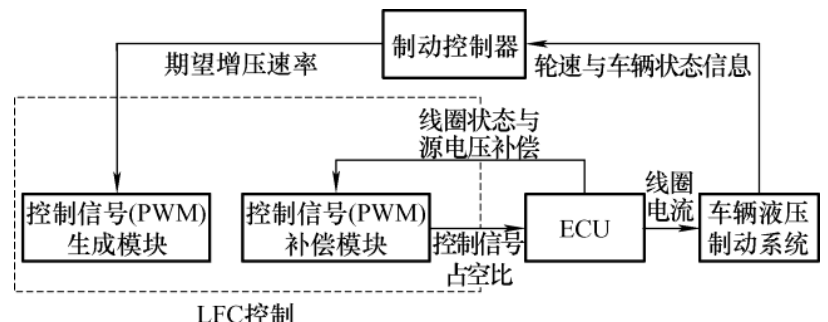

图 1 LFC 算法流程图
电磁阀状态方程, 首先采用平方根容积卡尔曼滤波 算法估计电磁阀阀芯行程, 准确计算出制动液流量 和轮缸压力, 然后采用滑模变结构方法控制阀芯行 程以实现制动压力的精确跟随。

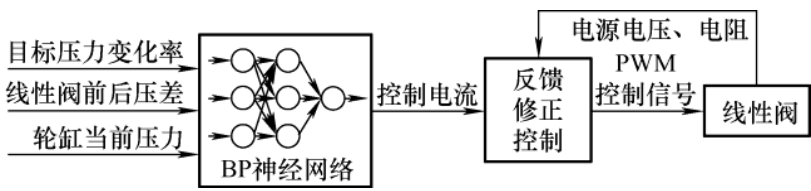

图 2 基于 $\mathrm{BP}$ 人工神经网络的线性阀压力控制流程图

液压制动系统存在非线性环节，如管路摩擦、 $p-V$ 特性等, 导致液压制动存在滞后性和惯性 ${ }^{[9]}$ 。不 同于液压制动系统的摩擦制动，电机制动采用的是 电磁制动, 二者在时域响应上存在较大差异, 且通 常液压制动的响应时间显著慢于电机制动。此外, 电机最大制动力矩受动力电池 SOC、电驱动系统温 度和驱动电机外特性等因素影响。因此, 若忽略电 机制动和液压制动系统的工作特性差异，则可能在 制动模式切换过程中产生较大的制动冲击, 降低车 辆平顺性及乘坐舒适性 ${ }^{[10]}$ 。

车辆行驶平顺性用于乘员在汽车行驶过程所处 的振动环境下具有的舒适度。对于车辆纵向平顺性 的研究, 众多学者以冲击度, 即加速度的导数作为 平顺性的评价指标 ${ }^{[1-12]}$ 。MUTOH 等 ${ }^{[11]}$ 基于制动减 速度和冲击度提出了 “不舒适指数” 用以描述制动平 顺性和舒适性。如图 3 所示, “不舒适指数” 可分为 五个等级, 且随制动减速度和冲击度的增大而增大。

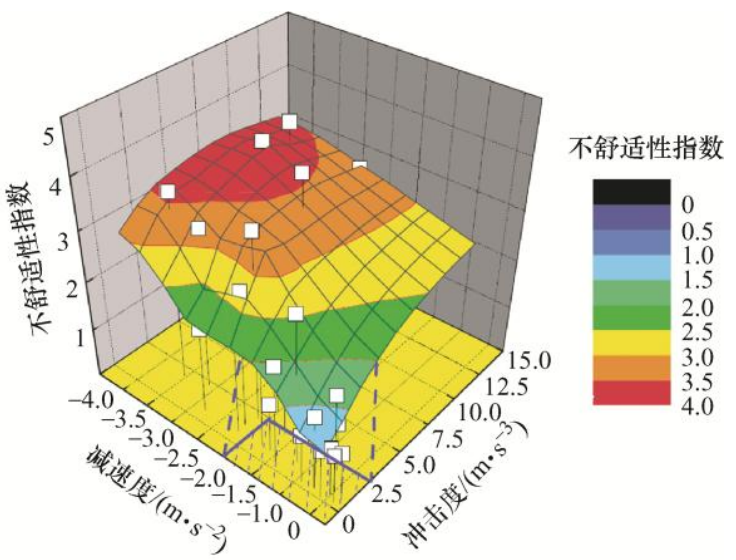

图 3 不舒适性指数与制动减速度、冲击度之间的关系

现有文献对电液复合制动系统的研究多集中在 电、液制动力分配, 较少考虑二者切换时的冲击及 其对制动平顺性的影响。电液复合制动过程中的冲 击主要存在于三种切换工况, 即电机制动力饱和时 液压制动介入、制动强度下降液压制动撤出以及低 速时电机制动撤出 ${ }^{[13]}$ 。现有文献提出的解决方案可 
归纳为如下四种: (1) 调节电机系统的响应速度, 使 其与液压制动系统趋于一致, 但该方法降低了系统 整体响应速度, 易造成制动滞后 ${ }^{[14]}$; (2) 提高液压 制动的响应速度, 但无法根除建压的滞后环节, 在 电机制动力饱和时控制效果欠佳 ${ }^{[15]}$; (3) 设计液压 启动预测控制, 消除液压制动延迟时间, 但不适用 于电子液压制动系统 ${ }^{[16]}$; (4)根据制动强度设计电机 制动力修正模块, 以解决液压制动介入时的冲击问 题, 但未考虑制动强度变化率, 易造成触发过频 ${ }^{[12]}$ 。 本文以轮毂电机驱动电动汽车电液复合制动系 统为研究对象, 采用分层控制架构, 上层控制器提 出了 “高压蓄能器+液压泵” 式 $\mathrm{EHB}$ 轮缸压力控制 方法; 针对电机制动与液压制动的时域响应差异及 车辆状态限制, 下层控制器设计了电液制动力分配 规则和切换工况下的平顺性控制策略; 最后, 基于 Simulink-AMESim 联合仿真平台验证了控制策略的 可行性。

\section{1 研究对象}

\section{1 电液复合制动系统}

如图 4 所示, 四轮轮毂电机驱动电动汽车电液 复合制动系统由动力电池系统、轮勃电机及其控制 器、“高压蓄能器+液压原”式 EHB 及整车控制器等 主要部件构成。整车控制器采集驾驶员制动意图及 车辆状态信息(如电池 SOC、电机系统温度、电机转 速等), 分配各轮处的电机制动与液压制动力, 电机 制动系统和液压制动系统执行制动请求完成行车 制动。

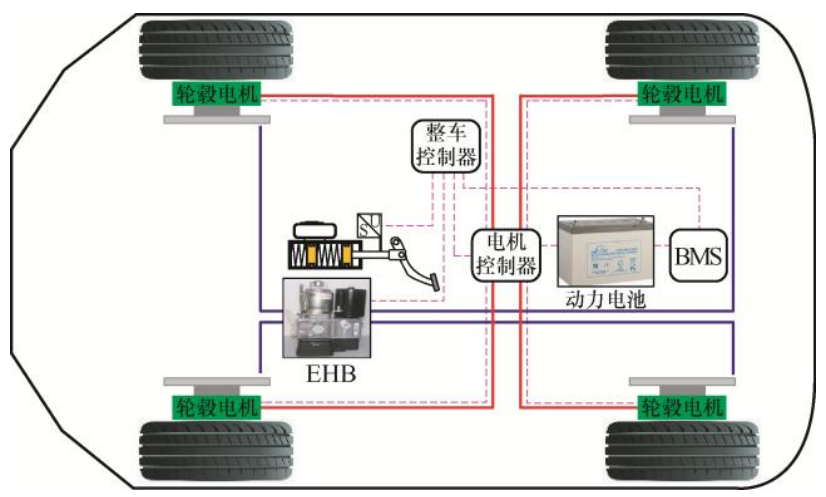

图 4 四轮轮勃电机驱动电动汽车电液复合制动系统

\section{2 电子液压制动系统}

电子液压制动系统拓扑结构如图 5 所示。电子 液压制动系统由制动踏板、液压执行单元、液压控 制单元以及盘式制动器等构成, 其中液压执行单元 的进液阀与出液阀为线性阀, 失效保护阀和平衡阀
为开关阀。通过控制各液压阀的状态, 可实现常规 制动、失效制动以及主动制动等功能。

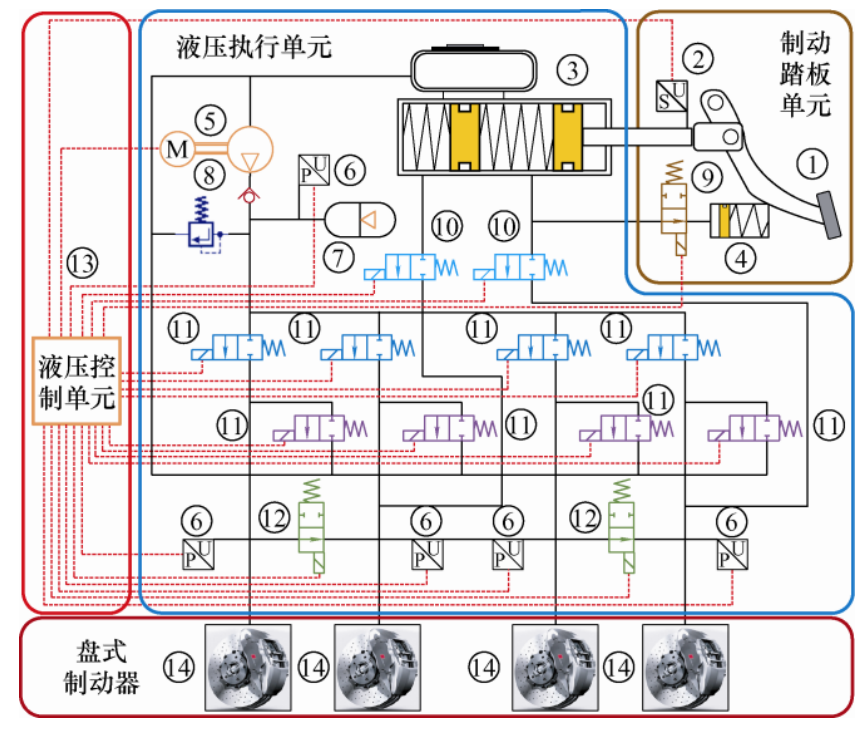

图 5 电子液压制动系统拓扑结构

(1) 制动踏板; (2) 踏板位移传感器; (3) 制动主缸; (4) 制动感觉模拟器; (5) 液压洜; (6) 压力传感器; (7) 高压蓄能器; (8) 溢流阀; (9) 制动感觉模拟器电磁威; (10) 失效保护阀; (11) 线性阀; (12) 平衡阀; (13) 液压控制单元; (14) 盘式制动器

\section{EHB 轮缸压力控制策略}

EHB 轮缸压力控制的实质为电磁阀控制, 首先 建立 EHB 系统各元件数学模型, 然后设计模糊控制 器控制轮缸进、出液线性阀的状态, 从而实现轮缸 压力的精确控制。

\subsection{EHB 液压元件建模}

高压蓄能器模型。EHB 蓄能器多选用皮囊式 高压蓄能器, 根据气体玻意耳定律, 其模型可表 示为

$$
P_{0} V_{0}^{n}=P_{\max } V_{1}^{n}=P_{\min } V_{2}^{n}=P V^{n}
$$

式中, $P_{0} 、 P_{\max } 、 P_{\min }$ 和 $P$ 分别为蓄能器初始充气 压力、最高工作压力、最低工作压力和当前工作压 力; $V_{0} 、 V_{1} 、 V_{2}$ 和 $V$ 分别为对应压力下的气体体积。

电机百模型。当高压蓄能器内压力下降至下限 阈值时, 电机泵开始工作直至高压蓄能器内压力升 至上限阈值, 其数学模型可表示为

$$
Q_{\text {out }}=V_{\mathrm{c}} S \frac{E}{E-\left[a p_{\text {in }}+(1-a) p_{\text {out }}\right]}
$$

式中, $Q_{\text {out }}$ 为电机原输出流量; $V_{\mathrm{c}}$ 为油原排量; $S$ 为电机转速; $E$ 为制动液体积模量; $p_{\text {in }}$ 为油原入口 端压力; $p_{\text {out }}$ 为油洜出口端压力; $a$ 为油厡压力因子。

进、出液线性阀模型。线性阀的流量特性与阀 
口的形状、前后压力差、通流截面积以及制动液的 密度、黏度、雷诺数等因素密切相关, 其数学模型 可表示为

$$
Q=C_{\mathrm{q}} A \sqrt{\frac{2|\Delta P|}{\rho}} \tanh \left(\frac{8 A}{\chi \eta_{0} \lambda_{\mathrm{c}}} \sqrt{\frac{2|\Delta P|}{\rho}}\right)
$$

式中, $Q$ 为线性阀处制动液流量; $C_{\mathrm{q}}$ 为最大流量系 数(其大小与阀口形状有关); $A$ 为节流孔截面积; $\Delta P$ 为阀口两端压力差; $\chi$ 为节流孔湿周长度; $\rho$ 为制动 液密度; $\eta_{0}$ 为制动液黏度; $\lambda_{\mathrm{c}}$ 为制动液雷诺数。

轮缸模型。如图 6 所示, 本文采用的是固定 钳盘式制动器, 其制动轮缸模型可简化为弹簧阻尼模型。根据工作特性, 轮缸工作过程可分为 两个阶段。

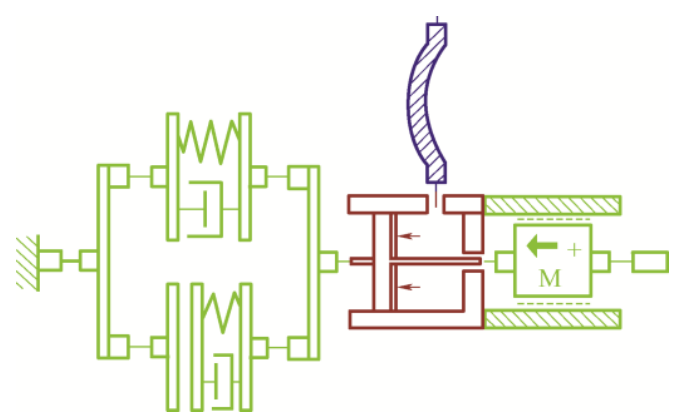

图 6 制动轮缸物理模型

第一阶段：液压推力主要用于克服制动盘与摩 擦片间的间隙 $\Delta x$, 该过程可表示为

$$
\left\{\begin{array}{l}
p \bullet A_{\mathrm{w}}=k_{\mathrm{cp}} \bullet x_{\mathrm{i}}+c_{\mathrm{cp}} \bullet \dot{x}_{\mathrm{i}}+m \bullet \ddot{x}_{\mathrm{i}} \\
x_{\mathrm{i}} \bullet A_{\mathrm{w}}=\int_{0}^{t} Q \mathrm{~d} t
\end{array}\right.
$$

式中, $p$ 为制动轮缸压力; $A_{\mathrm{w}}$ 为活塞截面积; $k_{\mathrm{cp}}$ 为 回位弹簧刚度; $x_{\mathrm{i}}$ 为活塞行程; $c_{\mathrm{cp}}$ 为活塞空程运动 阻尼; $m$ 为活塞组质量。

第二阶段：制动盘与摩擦片接触产生摩擦制动 力, 该过程可表示为

$$
\left\{\begin{array}{l}
p \bullet A_{\mathrm{w}}=k_{\mathrm{cp}} \bullet x_{\mathrm{i}}+\left(c_{\mathrm{cp}}+c_{\mathrm{i}}\right) \bullet \dot{x}_{\mathrm{i}}+k_{\mathrm{i}} \bullet\left(x_{\mathrm{i}}-\Delta x\right)+m \bullet \ddot{x}_{\mathrm{i}} \\
x_{\mathrm{i}} \bullet A_{\mathrm{w}}=\int_{0}^{t} Q \mathrm{~d} t
\end{array}\right.
$$

式中, $c_{\mathrm{i}}$ 为摩擦片与制动盘接触等效阻尼; $k_{\mathrm{i}}$ 为摩擦 片与制动盘接触等效刚度。

\section{2 基于模糊控制的 EHB 轮缸压力控制策略}

本文提出一种基于模糊控制的 $\mathrm{EHB}$ 轮缸压力 控制策略，其具体控制逻辑如图 7 所示。模糊控制 器的输入为轮缸输出压力与目标压力的偏差 $e$ 以及 偏差的变化率 $e_{\mathrm{c}}$, 输出为阀口面积的乘积系数 $u$, 即阀口开度。

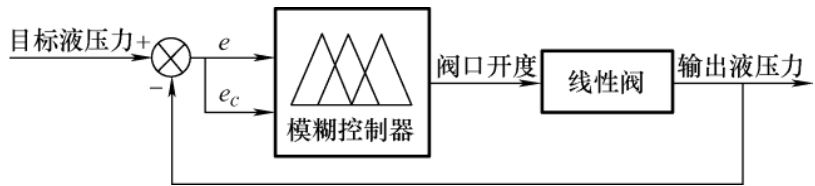

图 $7 \mathrm{EHB}$ 轮缸压力控制流程图

模糊控制器设计过程主要包括输入和输出变量 模糊化处理、隶属度函数确定和模糊控制规则制定 三个部分。

(1) 输入输出变量模糊化处理。根据制动系统 参数确定轮缸输出压力偏差的变化范围为 -13 $13 \mathrm{MPa}$, 偏差变化率的变化范围为 $-400 \sim$ $400 \mathrm{MPa} / \mathrm{s}$ 。进、出液阀输入及输出模糊化处理结

\begin{tabular}{|c|c|c|c|c|c|}
\hline $\begin{array}{l}\text { 类 } \\
\text { 别 }\end{array}$ & $\begin{array}{l}\text { 名 } \\
\text { 称 }\end{array}$ & $\begin{array}{l}\text { 基本 } \\
\text { 论域 }\end{array}$ & $\begin{array}{l}\text { 模糊 } \\
\text { 论域 }\end{array}$ & $\begin{array}{l}\text { 量化 } \\
\text { 因子 }\end{array}$ & 模糊语言集 \\
\hline 进 & $e$ & {$[0,13]$} & {$[0,13]$} & 1 & $\mathrm{ZO}, \mathrm{VS}, \mathrm{MS}, \mathrm{S}, \mathrm{B}, \mathrm{MB}, \mathrm{VB}$ \\
\hline 液 & $e_{\mathrm{c}}$ & {$[-400,0]$} & {$[-400,0]$} & 1 & $\mathrm{VB}, \mathrm{MB}, \mathrm{B}, \mathrm{S}, \mathrm{MS}, \mathrm{VS}, \mathrm{ZO}$ \\
\hline & $u_{\mathrm{i}}$ & {$[0,0.7]$} & {$[0,0.7]$} & 1 & $\mathrm{ZO}, \mathrm{VS}, \mathrm{MS}, \mathrm{S}, \mathrm{B}, \mathrm{MB}, \mathrm{VB}$ \\
\hline 出 & $e$ & {$[-13,0]$} & {$[0,13]$} & -1 & $\mathrm{ZO}, \mathrm{VS}, \mathrm{MS}, \mathrm{S}, \mathrm{B}, \mathrm{MB}, \mathrm{VB}$ \\
\hline 液 & $e_{\mathrm{c}}$ & {$[0,400]$} & {$[-400,0]$} & -1 & $\mathrm{VB}, \mathrm{MB}, \mathrm{B}, \mathrm{S}, \mathrm{MS}, \mathrm{VS}, \mathrm{ZO}$ \\
\hline 划 & $u_{\mathrm{o}}$ & {$[0,0.7]$} & {$[0,0.7]$} & 1 & $\mathrm{ZO}, \mathrm{VS}, \mathrm{MS}, \mathrm{S}, \mathrm{B}, \mathrm{MB}, \mathrm{VB}$ \\
\hline
\end{tabular}
果见表 1 。

表 1 进、出液阀输入与输出模糊化处理结果

（2）隶属度函数确定。为快速获得线性分析结 果, 降低控制系统的复杂度, 使用三角型函数作为输 入和输出的隶属度函数, 其隶属度分布如图 8 所示。

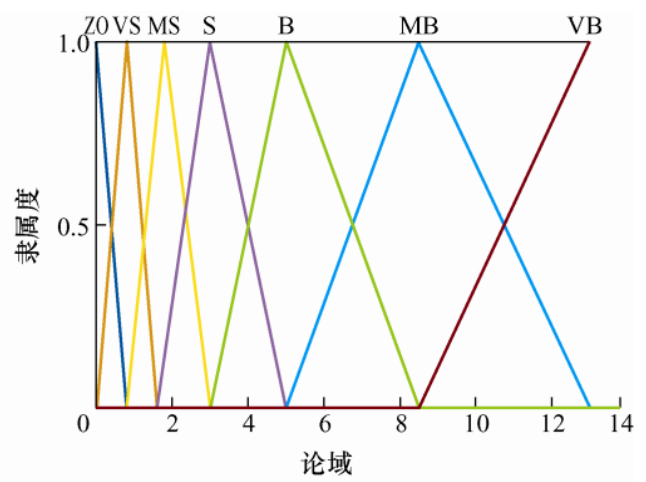

(a) $e$ 隶属度分布

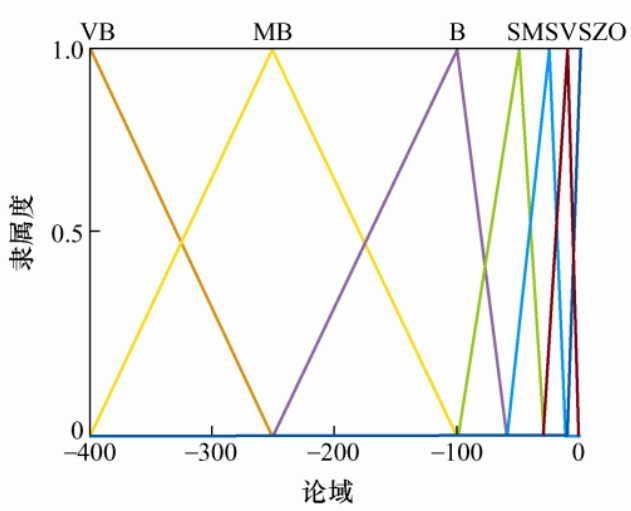

(b) $e_{\mathrm{c}}$ 隶属度分布 


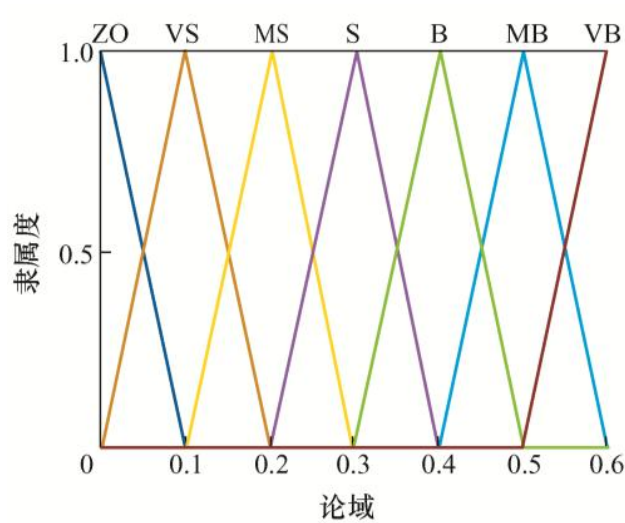

(c) $u_{\mathrm{i}}$ 隶属度分布

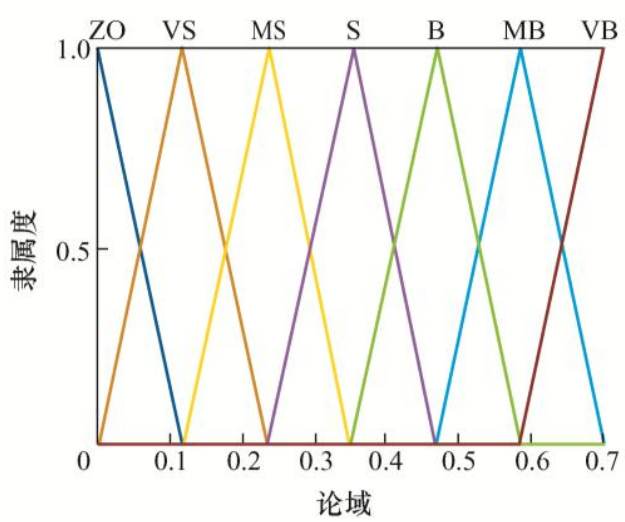

(d) $u_{\mathrm{o}}$ 隶属度分布

图 8 输入和输出隶属度分布图

(3) 模糊控制规则制定。由于轮缸的增压与 减压均为制动液由高压源流向低压源, 因此进、 出液阀可采用相同的模糊控制规则。但考虑到车 辆制动时动态载荷转移以及前后轴静载荷和制 动系统前、后轮轮缸参数存在差异, 因此对前、 后轮处线性阀分别建立模糊控制规则, 见表 2 和 表3。

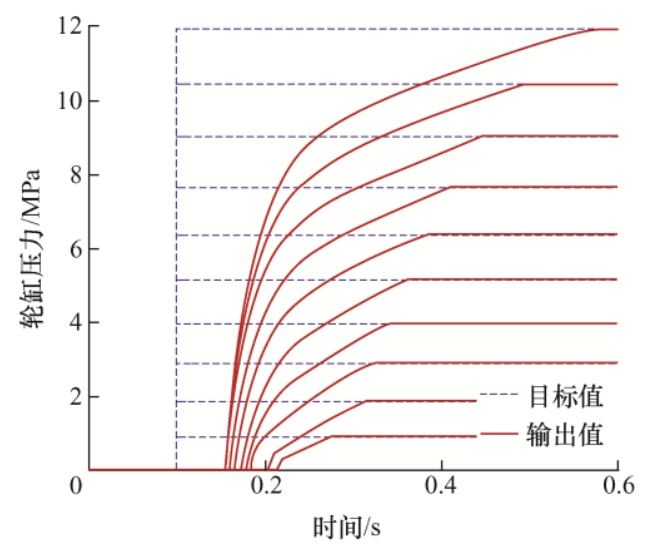

表 2 前轮线性阀模糊控制规则

\begin{tabular}{|c|c|c|c|c|c|c|c|}
\hline \multirow{2}{*}{$e$} & \multicolumn{7}{|c|}{$e_{\mathrm{c}}$} \\
\hline & VB & MB & B & $S$ & MS & VS & $\mathrm{ZO}$ \\
\hline $\mathrm{ZO}$ & MS & VS & $\mathrm{ZO}$ & $\mathrm{ZO}$ & $\mathrm{ZO}$ & $\mathrm{ZO}$ & $\mathrm{ZO}$ \\
\hline VS & MS & MS & $\mathrm{ZO}$ & $\mathrm{ZO}$ & $\mathrm{ZO}$ & VS & $\mathrm{S}$ \\
\hline MS & $S$ & VS & VS & VS & VS & VS & $\mathrm{S}$ \\
\hline$S$ & $S$ & $\mathrm{~S}$ & MS & MS & MS & MS & B \\
\hline B & B & B & $S$ & $S$ & S & $S$ & B \\
\hline MB & MB & MB & MB & MB & MB & MB & $\mathrm{MB}$ \\
\hline VB & VB & VB & VB & VB & VB & VB & $\mathrm{MB}$ \\
\hline \multicolumn{8}{|c|}{ 表 3} \\
\hline \multirow{2}{*}{$e$} & \multicolumn{7}{|c|}{$e_{\mathrm{c}}$} \\
\hline & VB & MB & B & $S$ & MS & VS & $\mathrm{ZO}$ \\
\hline $\mathrm{ZO}$ & MS & VS & $\mathrm{ZO}$ & $\mathrm{ZO}$ & $\mathrm{ZO}$ & $\mathrm{ZO}$ & $\mathrm{ZO}$ \\
\hline VS & S & MS & VS & VS & VS & $\mathrm{ZO}$ & S \\
\hline MS & $S$ & MS & VS & VS & VS & VS & $\mathrm{S}$ \\
\hline S & B & B & B & B & B & B & $\mathrm{MB}$ \\
\hline B & VB & VB & VB & VB & VB & VB & VB \\
\hline MB & VB & VB & VB & VB & VB & VB & VB \\
\hline VB & VB & VB & VB & VB & VB & VB & VB \\
\hline
\end{tabular}

\section{3 仿真验证}

搭建了 Simulink-AMESim 联合仿真平台, 仿真 车辆和 EHB 相关参数如表 4 所示。

表 4 车辆及 EHB 相关参数

\begin{tabular}{lc}
\hline \multicolumn{1}{c}{ 参数 } & 数值 \\
\hline 整备质量 $\mathrm{m} / \mathrm{kg}$ & 1740 \\
轴距 $l / \mathrm{mm}$ & 2500 \\
质心到前轴距离 $\mathrm{a} / \mathrm{mm}$ & 1150 \\
质心到后轴距离 $b / \mathrm{mm}$ & 1350 \\
质心高度 $h / \mathrm{mm}$ & 500 \\
蓄能器初始压力 $p_{0} / \mathrm{MPa}$ & 16 \\
前轮缸活塞直径 $d_{\mathrm{f}} / \mathrm{mm}$ & 45 \\
后轮缸活塞直径 $d_{\mathrm{r}} / \mathrm{mm}^{2}$ & 28 \\
进液阀阀口面积 $s_{\mathrm{in}} / \mathrm{mm}^{2}$ & 0.4 \\
出液阀阀口面积 $s_{\text {out }} / \mathrm{mm}^{2}$ & 0.6 \\
\hline
\end{tabular}

仿真工况包括制动强度 $z$ 增、减压阶跃输入工 况和正弦输入压力跟随工况。为简化计算, 前后轴 制动力按理想制动力分配曲线 $(I$ 曲线)分配, 仿真结 果如图 9 和图 10 所示。

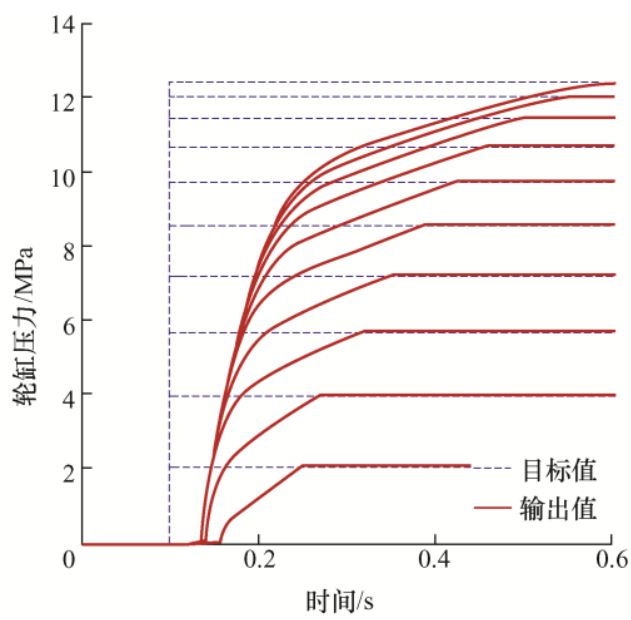

(a) 前、后轮轮㓡增压阶跃响应 $(z \in[0.1,1])$ 

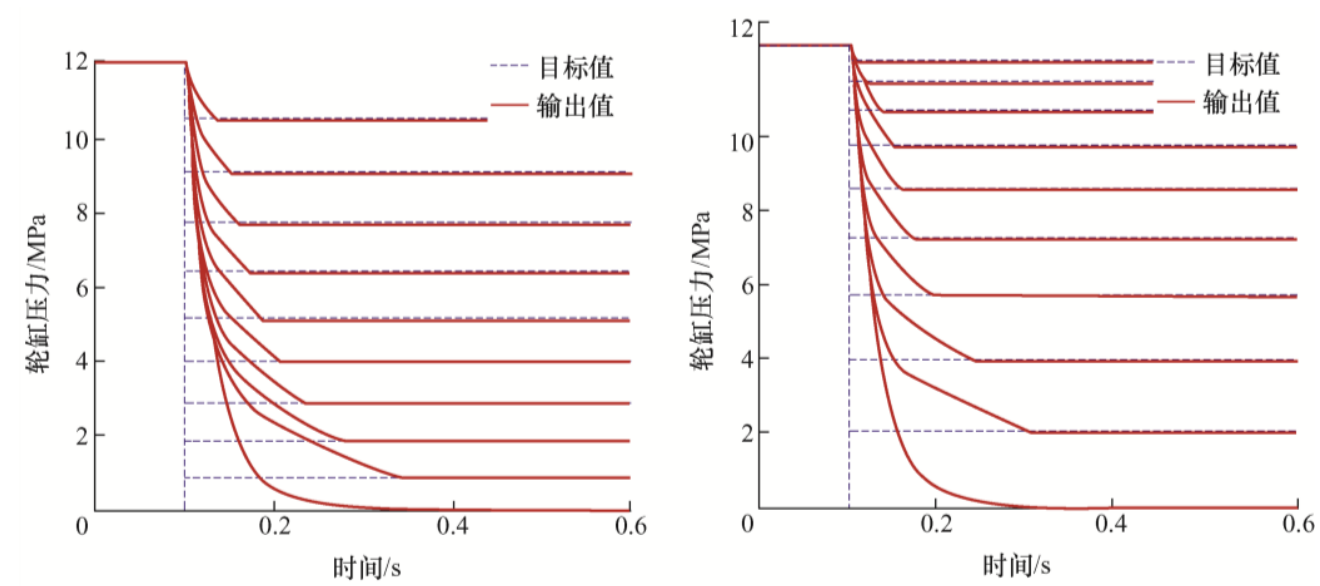

(b) 前、后轮轮缸减压阶跃响应 $(z \in[0,0.9])$

图 9 制动强度阶跃输入下的轮缸压力响应

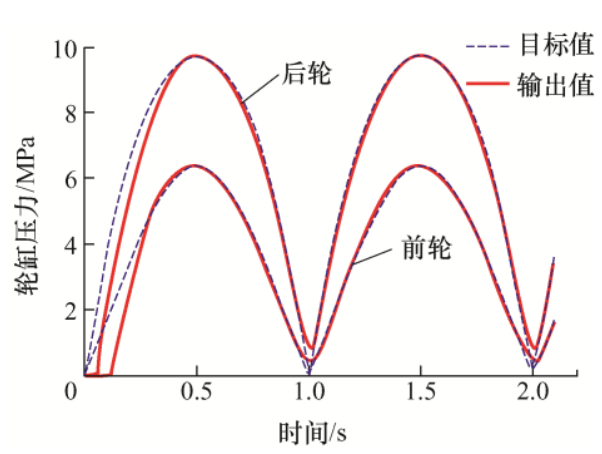

图 10 制动强度正弦输入下的轮缸压力响应

仿真结果表明, 在制动强度增、减压阶跃输入 下, 轮缸压力响应能在较短时间内达到稳态, 且稳 态误差不超过 $2 \%$; 在制动强度正弦输入下, 轮缸压 力响应能精确地跟随目标值。但由于液压系统 $p-V$ 特 性的影响, 导致轮缸压力响应出现较明显的滞后和惯 性环节, 因此, 可利用电机制动的快速响应特性进行 补偿控制, 以提高车辆制动平顺性与乘坐舒适性。

\section{3 电液复合制动力分配规则}

利用 EHB 的力学解耦特性, 按理想制动力分配 曲线 $(I$ 曲线)分配车辆前、后轴制动力, 前、后轴制 动力分配应满足

$$
F_{\mu 2}=\frac{1}{2}\left[\frac{G}{h_{g}} \sqrt{b^{2}+\frac{4 h_{g} l}{G} F_{\mu 1}}-\left(\frac{G b}{h_{g}}+2 F_{\mu 1}\right)\right]
$$

式中, $F_{\mu 1}$ 和 $F_{\mu 2}$ 分别为前、后轴制动力; $G$ 为汽车 总质量; $h_{g}$ 为质心高度; $l$ 为轴距; $b$ 为质心到后轴 的距离。

为提高制动能量回收率, 单轮电、液制动力分 配遵循电机制动为主、不足部分由液压制动补足的 原则。综合考虑动力电池 SOC、电机转速以及电机
制动力外特性等因素的影响, 制定电、液制动力分 配规则如图 11 所示。

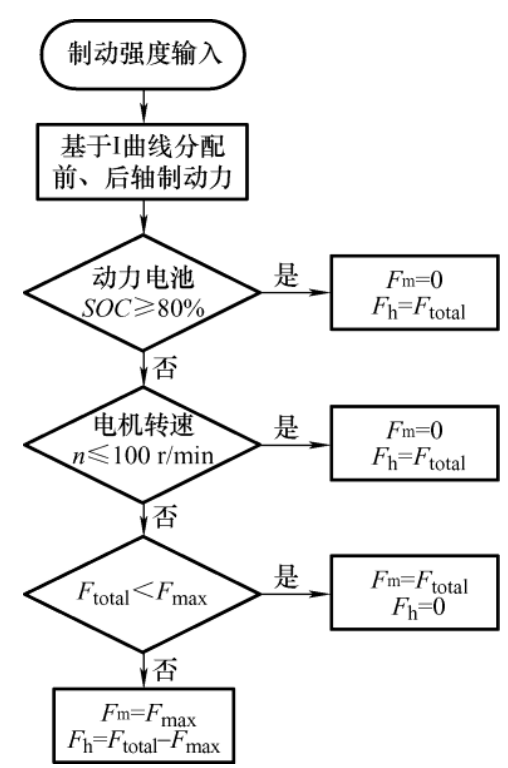

图 11 电、液制动力分配规则

$F_{\mathrm{m}} \square \square$ 电机制动力; $F_{\mathrm{h}} \square \square$ 液压制动力;

$F_{\text {total }} \square \square$ 单轮总制动力; $F_{\text {max }} \square \square$ 电机外特性上限制动力

\section{4 电液复合制动平顺性控制策略}

\section{1 控制策略}

由上文分析可知, 液压制动具有严重的滞后与 惯性特性，导致其时域响应速度显著低于电机制动， 进而引起电、液制动切换时的冲击, 影响制动舒适 性与安全性。由后文无控制仿真可知, 制动冲击主 要存在于电机制动力饱和时液压制动介入、低速时 电机制动撤出两个切换工况, 而制动强度下降过程 中液压制动撤出的切换工况无明显冲击。因此, 考 
虑电机制动和液压制动的响应差异, 本文提出一种 电液复合制动平顺性控制策略, 具体控制流程如 图 12 所示。该策略在实现电、液制动力分配和执行 功能的基础上增加了液压介入预测模块和电机制动 补偿模块。其中液压介入预测模块主要用于优化电 机制动力饱和时液压制动介入的切换工况, 而电机 制动补偿模块主要用于优化低速时电机制动撤出的 切换工况。

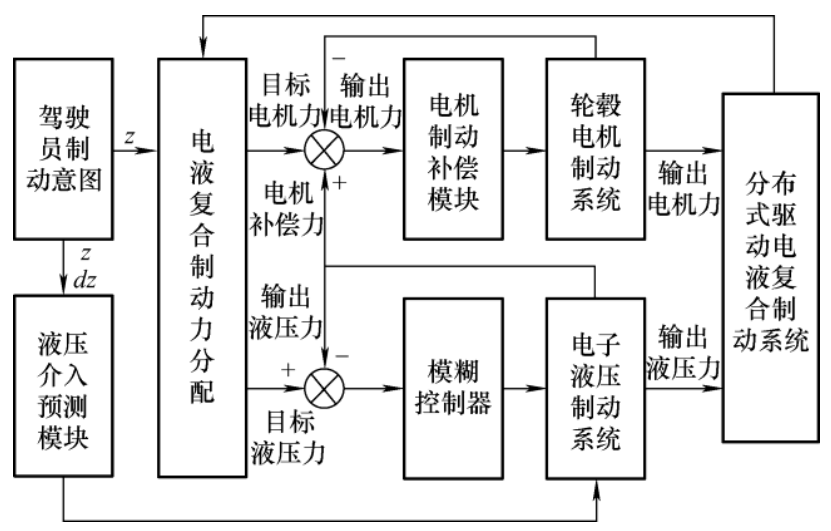

图 12 电液复合制动平顺性控制策略框图

\subsection{1 液压介入预测模块}

液压制动介入预测模块作用如下：根据制动强 度 $z$ 及其变化率 $\mathrm{d} z$ 判断驾驶员制动意图, 控制 $E H B$ 提前动作消除制动盘与摩擦片间隙 $\Delta x$, 以抵消 $p-V$ 特性延迟环节对制动轮缸建压过程的影响。

为避免制动时 $\mathrm{EHB}$ 的非必要启动, 引入制动强 度 $z$ 及其变化率 $\mathrm{d} z$ 作用如图 13 所示。针对不同制 动强度输入, 计算出不同的液压介入触发强度 $z_{\mathrm{cal}}$, 且满足

$$
z_{\text {cal }}=z_{\text {reg }}-\mathrm{d} z \cdot \Delta t
$$

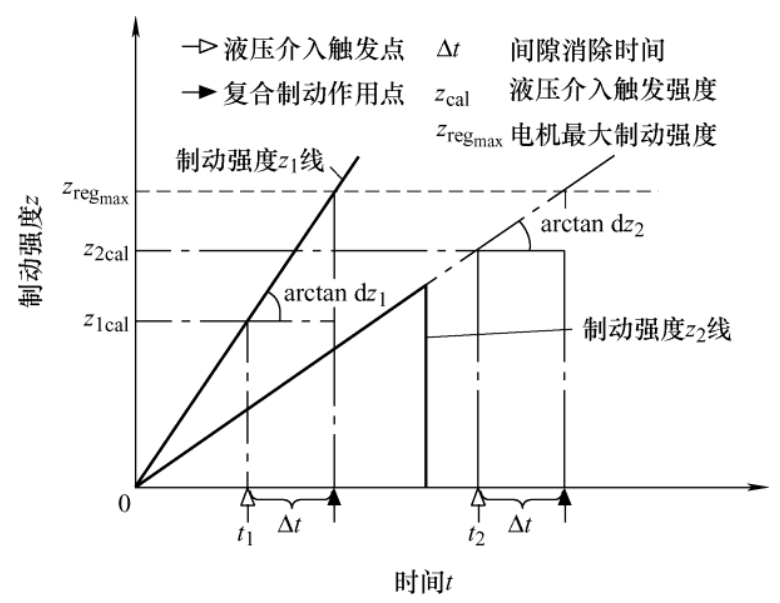

图 13 制动强度变化率 $d z$ 作用示意图

当制动强度 $z$ 超过液压介入触发强度 $z_{\mathrm{cal}}$ 时 (如 制动强度 $z_{1}$ 线), 触发液压介入预测模块, EHB 启 动消除制动间隙; 当制动强度 $z$ 未达到液压介入触
发强度 $z_{\mathrm{cal}}$ 且制动强度下降时(制动强度 $z_{2}$ 线), 则不 触发液压介入预测模块, EHB 无动作, 从而避免 $\mathrm{EHB}$ 非必要启动。

液压介入预测模块具体工作流程如图 14 所示。 驾驶员踩下制动踏板, ECU 根据踏板位移传感器采 集制动强度 $z$ 及其变化率 $\mathrm{d} z$, 同时计算液压介入触 发强度 $z_{\mathrm{cal}}$ 。当制动强度 $z$ 达到液压介入触发强度 $z_{\mathrm{cal}}$ 时, EHB 启动以消除摩擦片与制动盘间隙, 间隙消 除后, $\mathrm{EHB}$ 等待液压制动力分配。

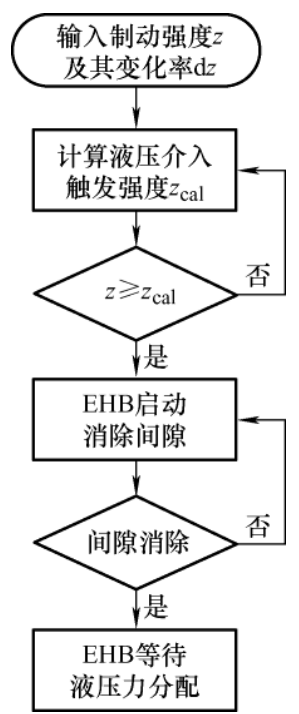

图 14 液压介入预测模块执行框图

\subsection{2 电动机制动补偿模块}

低速制动时, 由于轮毂电机的反电动势过低, 无法向动力电池回馈制动能量以及产生制动力, 此 时存在由电机制动向液压制动切换的工况。在此过 程中, 由于电机制动和液压制动的时域响应差异, 导致总制动力波动, 影响车辆平顺性。为此, 本文 提出一种基于 PID 矫正的电机制动补偿方法, 其具 体控制逻辑如图 15 所示。输入为修正目标电机制动 力矩与电机输出制动力矩的偏差, 输出为实际电机 输出制动力矩, 其中修正目标电机制动力为目标总 制动力与输出液压制动力的差值。

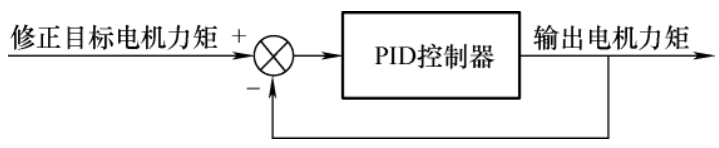

图 15 电机制动补偿模块执行框图

\section{2 仿真与验证}

搭建了 Simulink-AMEsim-Carsim 联合仿真平 台, 在电液复合制动三种典型切换工况下对提出的 平顺性控制策略进行了仿真验证。轮毂电机的具体 参数如表 5 所示, 其动态模型可近似为一阶惯性环 节, 电机转矩响应传递函数表示为 


$$
G(s)=\frac{1}{\tau s+1}
$$

表 5 轮毂电机参数

\begin{tabular}{lc}
\hline \multicolumn{1}{c}{ 参数 } & 数值 \\
\hline 电机基速 $n_{0} /(\mathrm{r} / \mathrm{min})$ & 700 \\
电机峰值功率 $P_{\mathrm{m}} / \mathrm{kW}$ & 23.5 \\
电机峰值转矩 $T_{\mathrm{m}} /(\mathrm{N} \cdot \mathrm{m})$ & 320 \\
低速临界转速 $n_{1} /(\mathrm{r} / \mathrm{min})$ & 80 \\
电机响应时间常数 $\tau / \mathrm{s}$ & 0.02 \\
\hline
\end{tabular}

仿真中, 车辆初始车速为 $50 \mathrm{~km} / \mathrm{h}$, 变制动强度 输入见图 16。在无控制、调节电机响应速度控制 ${ }^{[14]}$ 以及本文提出的平顺性控制策略下的仿真结果分别 如图 17 19 所示。

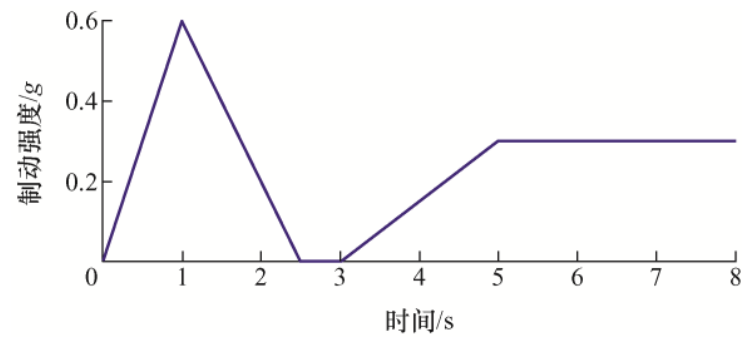

图 16 输入制动强度

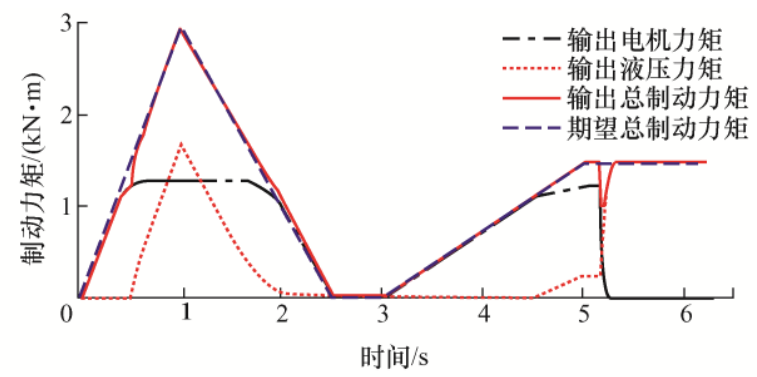

(a) 制动力矩

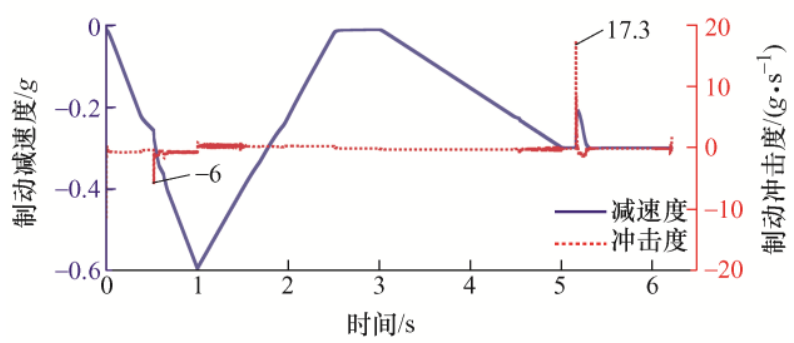

(b) 减速度与冲击度

图 17 无控制下的仿真结果

由图 $17 \mathrm{a}$ 可以看出, 在电机制动力饱和时液压 制动介入以及低速电机制动撤出的电液制动切换过 程中, 存在明显冲击。在无控制、调节电机响应速 度控制策略以及本文提出的平顺性控制策略下的总 制动力矩偏差及冲击度如表 6 所示。由仿真结果可 知, 文献[14]提出的调整电机响应速度的控制策略 虽能在一定程度上减小冲击度, 但输出总制动力矩 出现明显的滞后现象, 降低了电液复合制动系统的
响应速度。本文提出的电液复合制动平顺性控制策 略可以显著降低总制动力矩的最大跟随偏差，提高 了制动安全性，同时还能显著降低制动冲击，从而 有效提高了制动平顺性。

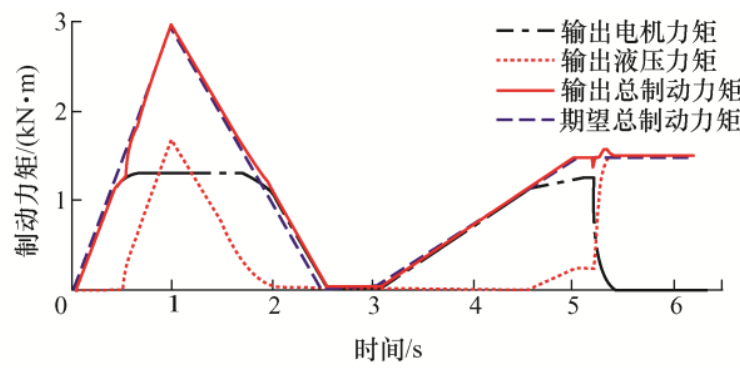

(a) 制动力矩

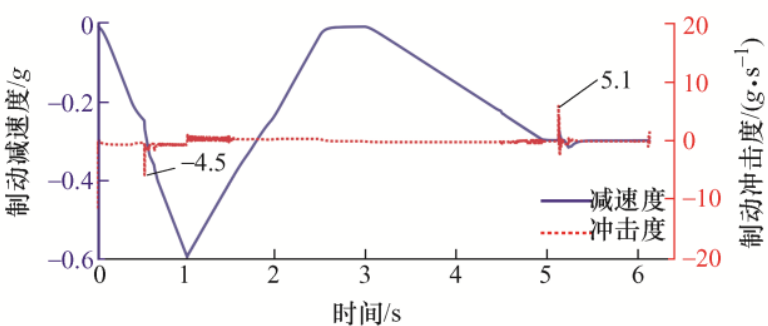

(b) 减速度与冲击度

图 18 调节电机响应速度控制策略下的仿真结果

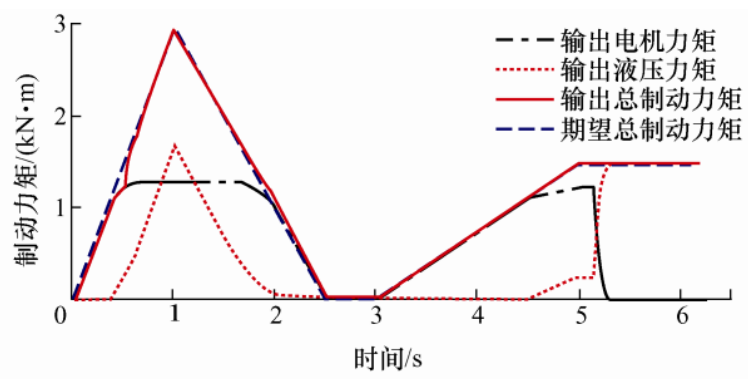

(a) 制动力矩

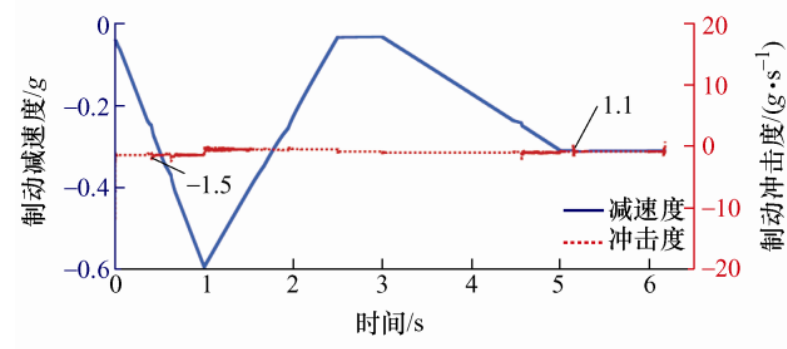

(b) 减速度与冲击度

图 19 本文提出的平顺性控制策略下的仿真结果

表 6 切换工况仿真结果

\begin{tabular}{cccc}
\hline \multirow{2}{*}{ 切换工况 } & 控制方法 & $\begin{array}{c}\text { 总制动力矩 } \\
\text { 最大偏差/(N·m) }\end{array}$ & $\begin{array}{c}\text { 冲击度 } \\
/\left(\mathrm{g} \cdot \mathrm{s}^{-1}\right)\end{array}$ \\
\hline \multirow{2}{*}{$\begin{array}{c}\text { 电机制动力饱和 } \\
\text { 时液压制动介入 }\end{array}$} & 无控制 & 295 & -6 \\
& 文献[14]控制策略 & 336 & -4.5 \\
& 本文控制策略 & 75 & -1.5 \\
\hline \multirow{2}{*}{ 低速时 } & 无控制 & 454 & 17.3 \\
电机制动撤出 & 文献[14]控制策略 & 102 & 5.1 \\
& 本文控制策略 & 18 & 1.1 \\
\hline
\end{tabular}




\section{5 结论}

(1) 针对 “高压蓄能器+电机百” 式电子液压制 动系统, 提出了基于模糊控制的轮缸压力控制策略, 该策略能够保证轮缸压力较好地追随目标液压力, 且稳态误差不超过 $2 \%$ 。

(2) 针对四轮轮㖕电机驱动电动汽车电液复合 制动系统, 提出了电液复合制动平顺性控制策略, 该策略保证了良好的复合制动响应，且显著降低了 制动模式切换过程中的冲击感, 提升了车辆制动平 顺性和乘坐舒适性。

\section{参 考 文 献}

[1] 熊璐, 钱超, 余卓平. 电动汽车复合制动系统研究现状 综述[J]. 汽车技术, 2015，2015(1): 1-8.

XIONG Lu, QIAN Chao, YU Zhuoping. Review on composite braking system of electric vehicle[J]. Automobile Technology, 2015, 2015(1): 1-8.

[2] WANG Z, ZHU J, ZHANG L, et al. Automotive ABS/DYC coordinated control under complex driving conditions[J]. IEEE Access, 2018, 2018(6): 32769-32779.

[3] XU W, CHEN H, ZHAO H, et al. Torque optimization control for electric vehicles with four in-wheel motors equipped with regenerative braking system[J]. Mechatronics, 2019, 2019(57): 95-108.

[4] 何仁, 冯海鹏. 自动紧急制动(AEB)技术的研究与进展 [J]. 汽车安全与节能学报, 2019，10(1)：5-19.

HE Ren, FENG Haipeng. Research and development of autonomous emergency brake (AEB) technology[J]. Automotive Safety and Energy，2019， 10(1): 5-19.

[5] 余卓平, 韩伟, 徐松云, 等. 电子液压制动系统液压力 控制发展现状综述 $[J]$. 机械工程学报, 2017，53(14): $1-15$.

YU Zhuoping, HAN Wei, XU Songyun, et al. Review on hydraulic pressure control of electro-hydraulic brake system[J]. Journal of Mechanical Engineering, 2017, 53(14): 1-15.

[6] CHOI S, LEE J, HWANG I. New generation ABS using linear flow control and motor speed control[J]. Journal of Passenger Car: Mechanical Systems Journal, 2003, 112(6): 337-342.

[7] 王祥. 电动轿车制动能量回收系统制动压力线性控制 算法研究[D]. 长春: 吉林大学, 2014.

WANG Xiang. Research on liner control algorithm for braking pressure of regenerative braking system on electric vehicle[D]. Changchun: Jilin University, 2014.

[8] 刘刚, 徐文博, 靳立强. 轮毂电机驱动电动汽车液压执 行单元的压力估计与控制方法研究 $[\mathrm{J}]$. 汽车工程, 2019, 41(10): 1138-1144.

LIU Gang, XU Wenbo, JIN Liqiang. A study on pressure estimation and control method for hydraulic actuation unit of hub-motor-driven electric vehicle[J]. Automotive Engineering, 2019, 41(10): 1138-1144.

[9] 熊璐, 韩伟, 余卓平, 等. 考虑关键非线性特征的集成 式电子液压制动系统主缸液压力精确控制 [J]. 机械工 程学报, 2019, 55(24): 117-126.

XIONG Lu, HAN Wei, YU Zhuoping, et al. Pressure precisely control of master cylinder on integrated-electro hydraulic brake system considering the critical nonlinear characteristics[J]. Journal of Mechanical Engineering, 2019, 55(24): 117-126.

[10] SUBRAMANIYAM K, SUBRAMANIAN S. Impact of regenerative braking torque blend-out characteristics on electrified heavy road vehicle braking performance[J]. Vehicle System Dynamics, 2019, DOI: https: //www. tandfonline.com/action/showCitFormats?doi=10.1080/00 423114.2019.1677921.

[11] MUTOH N, TAKITA K. A control method to suitably distribute electric braking force between front and rear wheels in electric vehicle systems with independently driven front and rear wheels[C]// Conference Record of the IAS Annual Meeting. Seattle : IEEE Industrial Applications Society, 2004, 2746-2753.

[12] 余卓平，史彪飞，熊璐，等. 集成式电子液压制动系统 的复合制动协调控制[J]. 同济大学学报, 2019, 47(6): 851-856.

YU Zhuoping, SHI Biaofei, XIONG Lu, et al. Coordination control of hybrid braking based on integrated-electro-hydraulic brake system[J]. Journal of Tongji University, 2019, 47(6): 851-856.

[13] CHIARA F, CANOVA M. A review of energy consumption, management, and recovery in automotive systems, with considerations of future trends[J]. Proceedings of the Institution of Mechanical Engineers, 2013, 227(6): 914-936.

[14] NUMASATO H, TOMIZUKA M. Settling control and performance of a dual-actuator system for hard disk drives[J]. IEEE/ASME transactions on mechatronics, 2003, 8(4): 431-438. 
[15] 朱智婷, 余卓平, 熊璐. 电动汽车复合制动系统过渡工 况协调控制策略 $[\mathrm{J}]$. 哈尔滨工程大学学报, 2014, 35(9): 1135-1141.

ZHU Zhiting, YU Zhuoping, XIONG Lu. Coordination control strategy of electric vehicle hybrid brake system in transient conditions $[\mathrm{J}]$. Journal of Harbin Engineering University, 2014, 35(9): 1135-1141.

[16] 付晓丹, 罗禹贡, 韩云武, 等. 智能混合动力汽车电液 复合制动的协调控制策略 [J]. 汽车工程, 2011, 33(11): 915-919.

FU Xiaodan, LUO Yugong, HAN Yunwu, et al.
Coordinated control strategy for the electro-hydraulic braking system of intelligent hybrid electric vehicles[J]. Automotive Engineering, 2011, 33(11): 915-919.

作者简介: 张雷, 男, 1987 年出生, 博士, 长聘副教授, 硕士研究生导 师。主要研究方向为智能网联新能源汽车整车动力学控制及储能系统管 理技术等。

E-mail: lei_zhang@bit.edu.cn

刘青松, 男, 1995 年出生。主要研究方向为分布式驱动电动汽车动力学 控制。

Email: kg609521490@163.com

王震坡(通信作者), 男, 1976 年出生, 博士, 教授, 博士研究生导师。 主要研究方向为电动汽车动力学理论与控制以及车用锂离子动力电池 成组理论与技术。

Email: wangzhenpo@bit.edu.cn 